\title{
Photoacoustics resolves species-specific differences in hemoglobin concentration and oxygenation
}

\author{
Lina Hacker, ${ }^{\text {a,b }}$ Joanna Brunker, ${ }^{\text {a,b }}$ Ewan St. John Smith, ${ }^{\mathrm{c}}$ \\ Isabel Quiros-Gonzalez, ${ }^{\mathrm{a}, \mathrm{b}, *, \dagger}$ and Sarah E. Bohndiek ${ }^{\mathrm{a}, \mathrm{b}, *}$ \\ ${ }^{a}$ University of Cambridge, Department of Physics, Cambridge, United Kingdom \\ ${ }^{b}$ University of Cambridge, Cancer Research UK Cambridge Institute, Cambridge, \\ United Kingdom \\ ${ }^{\mathrm{c}}$ University of Cambridge, Department of Pharmacology, Cambridge, United Kingdom
}

\begin{abstract}
Significance: Photoacoustic imaging (PAI) enables the detection of blood hemoglobin (HB) concentration and oxygenation $\left(\mathrm{sO}_{2}\right)$ with high contrast and resolution. Despite the heavy use of photoacoustically determined total hemoglobin $(\mathrm{THb})$ and oxygenation $\left(\mathrm{sO}_{2}\right)$ biomarkers in PAI research, their relationship with underlying biochemical blood parameters and the impact of intra- and interspecies genetic variability have yet to be established.
\end{abstract}

Aim: To explore the relationship between $\mathrm{THb}$ and $\mathrm{sO}_{2}$ photoacoustic biomarkers and the underlying biochemical blood parameters in a species-specific manner.

Approach: Experiments were performed on blood in vitro using tissue-mimicking agar phantoms. Blood was extracted from mouse, rat, human, and naked mole-rat (Heterocephalus glaber), anticoagulated in ethylenediaminetetraacetic acid, and measured within $48 \mathrm{~h}$. THb and $\mathrm{sO}_{2}$ were measured using a commercial photoacoustic tomography system (InVision 128, iThera Medical $\mathrm{GmBH})$. Biochemical blood parameters such as $\mathrm{HB}$ concentration $(\mathrm{g} / \mathrm{dL})$, hematocrit (HCT, \%), and red blood cell (RBC) count $\left(\mu \mathrm{L}^{-1}\right)$ were assessed using a hematology analyzer (Mythic 18 Vet, Woodley Equipment).

Results: A significant correlation was observed between THb and biochemical HB, HCT, and $\mathrm{RBC}$ in mouse and rat blood. Moreover, PAI accurately recapitulated interspecies variations in HB and HCT between mouse and rat blood and resolved differences in the oxygen dissociation curves measured using $\mathrm{sO}_{2}$ between human, mouse, and rat. With these validation data in hand, we applied PAI to studies of blood obtained from naked mole-rats and could confirm the high oxygen affinity of this species in comparison to other rodents of similar size.

Conclusions: Our results demonstrate the high sensitivity of photoacoustically determined hemoglobin biomarkers toward species-specific variations in vitro.

(C) The Authors. Published by SPIE under a Creative Commons Attribution 4.0 Unported License. Distribution or reproduction of this work in whole or in part requires full attribution of the original publication, including its DOI. [DOI: 10.1117/1.JBO.25.9.095002]

Keywords: photoacoustic imaging; hemoglobin; phantom; oxygenation.

Paper 200056RR received Mar. 3, 2020; accepted for publication Aug. 11, 2020; published online Sep. 4, 2020.

\section{Introduction}

Photoacoustic imaging (PAI) is an emerging modality able to reveal high image contrast, arising from optical absorption in tissue at high spatial resolution, afforded by ultrasound detection. PAI is based on the photoacoustic principle, ${ }^{1}$ whereby pulsed light is absorbed by chromophores resulting in the generation of pressure waves that can be detected by ultrasound transducers

\footnotetext{
*Address all correspondence to Isabel Quiros-Gonzalez, E-mail: Isabel.QuirosGonzalez@cruk.cam.ac.uk; Sarah E. Bohndiek, E-mail: seb53@cam.ac.uk

${ }^{\dagger}$ Present address: University of Oviedo, IUOPA, and FINBA, School of Medicine, Morphology and Cell Biology Department, Julian Claveria, Spain
} 
at the tissue surface. Applying PAI at multiple wavelengths enables noninvasive, label-free detection of total hemoglobin concentration $(\mathrm{THb})$ and oxygenation $\left(\mathrm{sO}_{2}\right)$ based on the different optical absorption spectra of deoxygenated $(\mathrm{HbR})$ and oxygenated hemoglobin $\left(\mathrm{HbO}_{2}\right)^{2}$. The relative concentrations of these respective chromophores can then be calculated by spectroscopic inversion. ${ }^{3}$ PAI measures of $\mathrm{THb}\left(\mathrm{HbR}+\mathrm{HbO}_{2}\right)$ and $\mathrm{sO}_{2}\left(\mathrm{HbO}_{2} / \mathrm{THb}\right)$ have been widely exploited to characterize tissues in the context of different pathologies, for example in breast cancer, ${ }^{4-6}$ melanomas, ${ }^{7,8}$ prostate cancer, ${ }^{9-11}$ nodal lesions of the head and neck, ${ }^{12}$ vascular diseases, ${ }^{13-15}$ and colitis. ${ }^{16}$ Particularly in cancer biology, $\mathrm{THb}$ and $\mathrm{sO}_{2}$ have proved to be of high value by allowing the detection of two cancer hallmarks: angiogenesis and hypoxia. ${ }^{17}$

Despite their extensive use in PAI, the relationship between photoacoustically determined $\mathrm{THb}$ and $\mathrm{sO}_{2}$ biomarkers and the underlying physiological variations in biochemical blood parameters, such as hemoglobin (HB) concentration (g/dL), hematocrit (HCT, \%), and red blood cell (RBC) count $\left(\mu \mathrm{L}^{-1}\right)$, has yet to be established. HB is an iron-containing hem tetramer composed of two $\alpha$ - and two $\beta$-monomers and responsible for oxygen transport in all vertebrates, ${ }^{18}$ yet HB-related blood parameters differ within and between species and human race, ${ }^{19,20}$ with age and $\operatorname{sex}^{21-23}$ of the individual, and can change under pathophysiological conditions or pharmaceutical treatment. ${ }^{24}$ Moreover, genetic modifications of the HB protein sequence resulting from either accumulated evolutionary changes or spontaneous mutations can alter the oxygen binding capabilities of the HB molecule. ${ }^{25-27}$ Such differences could lead to substantial variations in PAI biomarkers or, for example, signal dynamics during more complex PAI protocols such as a gas challenge, ${ }^{10}$ which could lead to misinterpretation of PAI data.

Here, we investigate the relationship of photoacoustically determined $\mathrm{THb}$ and $\mathrm{sO}_{2}$ biomarkers with biochemical blood parameters taken from mice, rats, and humans in a controlled tissue-mimicking phantom system. Having examined the intraspecies homogeneity of the globin genes, we first elucidate intra- and interspecies differences between photoacoustic $\mathrm{THb}$ and static biochemical blood parameters in mouse and rat. We then move on by examining the differences in the dynamics of the oxygen dissociation curves (ODCs), here including human as a further species. With these validation data in hand, we then apply PAI to studies of blood obtained from naked mole-rats (Heterocephalus glaber), a species remarkable for its resistance to oxygen deprivation. ${ }^{28}$ Our results confirm the high sensitivity of photoacoustically determined biomarkers toward species-specific variations in vitro, which should be considered in future study designs.

\section{Materials and Methods}

\subsection{Sequencing}

Deoxyribose nucleic acid (DNA) was isolated from liver samples of female C57BL/6J mice (3 to 4 months, $n=3$ ) and Wistar rats (6 to 9 months, $n=3$ ) (Charles River) using the Qiagen DNeasy Blood/Tissue kit (Cat. no. 69504) following the manufacturer's instruction. Primers (displayed in Table 1) for both the forward and reverse strand of each gene were designed using Primer 3 software (version 4.0.0). ${ }^{29}$ Polymerase chain reaction (PCR) amplification was performed using the Q $5^{\circledR}$ Hot Start High-Fidelity DNA Polymerase (New England BioLabs Inc.) and the corresponding protocol ${ }^{30}$ with an annealing temperature of $66^{\circ} \mathrm{C}$. Products were purified and sequenced using Sanger sequencing (SourceBioscience). Sequences were aligned using Clustal Omega. ${ }^{31}$ The identity match between the sequences was calculated.

\subsection{Blood Samples}

Whole blood and tissue samples (liver) for DNA extraction originating from female C57BL/6J mice (3 to 4 months) and Wistar rats (6 to 9 months) were ordered from Charles River Laboratories. For comparison of the blood parameters in Secs. 1-3, the same sex was chosen as HB parameters have been shown to be sensitive to the sex of individuals. ${ }^{22}$ For the blood oxygenation experiments in the remaining Secs. 4 and 5, blood from mixed sex was used to achieve more generalizable results. Human blood samples from healthy donors were collected 
Table 1 Confirmed intraspecies gene sequence identity of $\mathrm{HB} \alpha$ - and $\beta$-genes in female mouse $(n=3)$ and rat $(n=3)$.

\begin{tabular}{|c|c|c|c|c|}
\hline Species & Gene & Primer sequence & & $\begin{array}{l}\text { Intraspecies gene } \\
\text { identity (\%) }\end{array}$ \\
\hline \multirow[t]{10}{*}{ Mouse } & HBA-A1 & Exon $1+2$ & F: GGGCAACTGATAAGGATTCCC & 100 \\
\hline & & & R: GACCACTATGTTCCСTGCCT & \\
\hline & & Exon 3 & F: TGTCCACTTTGTCTCCGCA & 100 \\
\hline & & & R: ACATGACACCTTTGCAGACG & \\
\hline & HBA-A2 & Exon $1+2$ & F: CTACTTGCTGCAGGTCCAA & 100 \\
\hline & & & R: CCAGGTCCCAGCGCATAC & \\
\hline & & Exon 3 & F: TGTCCACTTTGTCTCCGCA & 100 \\
\hline & & & R: AGAAGCGTCCCCACACTAAA & \\
\hline & HBB-t & Exon $1+2$ & F: TCATCTCTGAAGCCTCACCC & 100 \\
\hline & & & R: ATAGCCAGGGGAAGGAAACC & \\
\hline \multirow[t]{8}{*}{ Rat } & HBA-A1 & Exon $1+2$ & F: GAAACTTGCTGCAGGGTCAA & 100 \\
\hline & & & R: GCCAGGTCTGAGCTCACA & \\
\hline & HBA-A2 & Exon $1+2$ & F: CAATGACAGCTGCTCCAAGG & 100 \\
\hline & & & R: CAAGGGATCTCTGGAGGACC & \\
\hline & HBA-A3 & Exon $1+2$ & F: GCTGCAGGGCCAATACATTC & 100 \\
\hline & & & R: GCCAGGTCTGAGCTCACA & \\
\hline & HBB & Exon $1+2$ & F: ATTGGCCAATCTGCTCACAC & 100 \\
\hline & & & R: GAAAGCCACAGGAAGGACAC & \\
\hline
\end{tabular}

under the research ethics approval of the Royal Papworth Hospital tissue bank (project number T02196) in Cambridge. Blood from naked-mole rats (15 to 23 months, all male) was collected as a postmortem nonregulated procedure following decapitation of the animal for another scientific purpose. All blood samples were anticoagulated in ethylenediaminetetraacetic acid, directly stored at $4^{\circ} \mathrm{C}$, and processed within $48 \mathrm{~h}$.

\subsection{Blood Analysis}

Blood parameters were assessed using an impedance-based hematology analyzer (Mythic 18, Woodley Veterinary Diagnostics ${ }^{32}$ ). The parameters obtained included: absolute and relative number of lymphocytes, monocytes, and granulocytes; absolute number of $\mathrm{RBC}\left(\mu \mathrm{L}^{-1}\right)$ and white blood cells (WBC, $\mu \mathrm{L}^{-1}$ ); HB concentration ( $\mathrm{g} / \mathrm{dL}$ ); mean corpuscular hemoglobin ( $\mathrm{MCH}, \mathrm{pg}$ ); mean corpuscular volume $\left(\mathrm{MCV}, \mu \mathrm{m}^{3}\right)$; mean corpuscular hemoglobin concentration (MCHC, $\mathrm{g} / \mathrm{dL}$ ); and $\mathrm{HCT}(\%)$. MCV, MCHC, and $\mathrm{MCH}$ are commonly defined as: $\mathrm{MCV}=$ $[\mathrm{HCT}] / \mathrm{RBC}, \mathrm{MCHC}=[\mathrm{HB}] /[\mathrm{HCT}]$, and $\mathrm{MCH}=[\mathrm{HB}] / \mathrm{RBC}$, where square brackets denote concentrations.

\subsection{Absorption Spectra Measurement}

The absorption profile of HB was independently verified. Whole blood was lysed in distilled water $(1: 1)$ and the HB extracted in order to avoid artifacts caused by light scattering of the erythrocytes. For HB extraction, ${ }^{33}$ blood was centrifuged three times at $3000 \mathrm{rpm}$ for 3 min and washed with phosphate buffered saline (PBS) to remove the plasma. Afterward, 
1 unit volume of blood was thoroughly mixed with 1 unit volume of deionized water and 0.4 unit volume of toluene. The mixture was stored at $4^{\circ} \mathrm{C}$ for at least $24 \mathrm{~h}$ to ensure complete hemolysis. The solution was then centrifuged at $13,000 \mathrm{rpm}$ for $10 \mathrm{~min}$. The lowest layer containing the HB was extracted by syringe and filtered through a syringe filter (Millex, Millipore) with a pore size of $0.22 \mu \mathrm{m}$ to remove the cell debris and large particles. The absorption spectrum of extracted HB was recorded in the range of 600 to $900 \mathrm{~nm}$ using a microplate reader (Clariostar, BMG Labtech). The absorption spectra were normalized to the area under the curve to account for the different HB levels of rat and mouse blood.

\subsection{Phantom Preparation and Photoacoustic Imaging}

Agar phantoms were produced according to the protocol by Joseph et al. ${ }^{36}$ Briefly, liquid $1.5 \%$ w/v agar (Fluka 05039) was mixed with $2.1 \%$ v/v prewarmed intralipid (Sigma-Aldrich I141) to provide a reduced scattering coefficient of $5 \mathrm{~cm}^{-1}$. Nigrosin dye (Sigma-Aldrich 198285) was added to provide an absorption coefficient of $0.05 \mathrm{~cm}^{-1}$ (at $564 \mathrm{~nm}$, peak of the nigrosin spectrum). The solution was poured into a $20-\mathrm{mL}(2-\mathrm{cm}$ diameter) syringe with the injection end removed and with polyvinyl chloride tubing (inner diameter: $1.5 \mathrm{~mm}$, outer diameter: $2.1 \mathrm{~mm}$; VWR 228-3857) inserted along the central axis. After the agar was set, the phantom was removed from the syringe ready for imaging.

PAI experiments were performed using a commercial small animal imaging system (MSOT inVision 256-TF; iThera Medical GmbH). The system has been described in detail elsewhere. ${ }^{37,38}$ Briefly, a tunable (660 to $1300 \mathrm{~nm}$ ) optical parametric oscillator (OPO), pumped by a nanosecond (ns) pulsed $\mathrm{Nd}$ :YAG laser, with $10-\mathrm{Hz}$ repetition rate and up to 7 -ns pulse duration provides excitation pulses. Light is delivered to the sample through a custom optical fiber assembly, which creates a diffuse ring of uniform illumination over the imaging plane. The sample is coupled to the transducers using a water bath, filled with degassed, and deionized water. For ultrasound detection, 256 toroidally focused ultrasound transducers covering an angle of $270 \mathrm{deg}$ are used (center frequency of $5 \mathrm{MHz}, 60 \%$ bandwidth) allowing tomographic reconstruction. A minimum of four images were taken along the length of the phantom at steps of $0.5 \mathrm{~mm}$ using seven wavelengths $(700,730,760,800,850,900$, and $1040 \mathrm{~nm})$ with an average of 10 pulses per wavelength.

For the measurements of the ODC, a flow phantom set up was used, which has also been described in detail elsewhere. ${ }^{39}$ For each measurement, $5 \mathrm{~mL}$ of pooled blood from the respective species was first oxygenated by the addition of $0.2 \% \mathrm{v} / \mathrm{v}$ hydrogen peroxide $\left[\mathrm{H}_{2} \mathrm{O}_{2} 30 \%(\mathrm{w} / \mathrm{w})\right.$ in deionized water, Sigma-Aldrich 7722-84-1]. The oxygenated blood was filled into the circuit, carefully avoiding the introduction of air bubbles. During the course of the experiment, a syringe driver (Harvard, MKCB2159V) was used to deoxygenate the blood with $0.03 \% \mathrm{w} / \mathrm{v}$ sodium hydrosulfite (ACROS Organics 7775-14-6) in PBS) at a constant flow rate of $10 \mu \mathrm{L} / \mathrm{min}$. The experiment was performed at room temperature and a peristaltic pump (Fisher Scientific CTP100) was used for blood circulation. Oxygen fluorescence quenching needle probes (Oxford Optronix, NX-BF/O/E) were placed before and after the tissue-mimicking phantom, which recorded the temperature and partial pressure of oxygen $\left(\mathrm{pO}_{2}, \mathrm{mmHg}\right)$ in real time. The data were downloaded via an Arduino UNO and read in MATLAB ${ }^{\circledR}$. Using the same commercial PAI system as above, images were acquired at a single position (no pulse-to-pulse averaging) for 17 wavelengths $(660,664,680,684,694,700,708,715,730,735,760,770,775,779$, 800,850 , and $950 \mathrm{~nm}$ ). Absorption spectra were measured using a light source (Avantes Avalight-HAL-S-Mini) and spectrometer (AvaSpec-ULS2048-USB2-VA-50). Absorption spectra were recorded continuously via AvaSoft software as the fluid passed through a flow cell (Hellma Analytics, 170700-0.5-40) as it has been shown that directly measured absorption spectra provide the most accurate endmembers for spectral unmixing. ${ }^{39}$ Species-specific absorption spectra at the point of complete oxygenation and deoxygenation were extracted and used for spectral unmixing of the data recorded for the respective species. Between each measurement run, the tubing containing the blood was cleaned with PBS.

For the experiments involving naked mole-rat blood, a simpler set up was used as only a limited amount of naked mole-rat blood could be obtained. Blood samples (100 $\mu \mathrm{L})$ were inserted into a straw within a phantom and $10 \mu \mathrm{L} \mathrm{H}_{2} \mathrm{O}_{2}(0.03 \%$ in PBS) was injected using 
a syringe. The oxygenation of the blood was directly measured after injection using MSOT. Thirty images were taken of a chosen slice using 7 wavelengths $(700,730,760,800,850$, 900 , and $1040 \mathrm{~nm}$ ), with an average of 10 pulses per wavelength. Each image took $7 \mathrm{~s}$ to acquire.

Measurements were conducted at $37^{\circ} \mathrm{C}$. It should be noted here that naked-mole rats are considered poikilothermic and usually have a physiological body temperature of around $30^{\circ} \mathrm{C}$ to $32^{\circ} \mathrm{C}^{40}$ Examining the oxygen affinity at higher temperature decreases the oxygen affinity of the blood. ${ }^{41}$ However, it has been shown that at $37^{\circ} \mathrm{C}$ significant differences between the oxygen affinity of naked mole-rat and mouse are still present, ${ }^{42}$ supporting our experimental approach.

\subsection{Image and Statistical Analysis}

PAI data were analyzed using the ViewMSOT software (v3.6.0.119; iThera Medical GmbH). Model-based image reconstruction and linear multispectral processing were applied to extract the relative signal contributions of $\mathrm{HbO}_{2}$ and $\mathrm{HbR}$. The same position within the phantom was used to determine the average intensities for $\mathrm{HbO}_{2}$ and $\mathrm{HbR}$. Regions of interest (ROIs) were manually drawn around the circular cross section of the tube inserted in the phantom. $\mathrm{THb}^{\mathrm{MSOT}}$ was calculated as the sum of $\mathrm{HbO}_{2}$ and $\mathrm{HbR}$. $\mathrm{sO}_{2}^{\mathrm{MSOT}}$ was calculated as the ratio of $\mathrm{HbO}_{2}$ to $\mathrm{THb}^{\mathrm{MSOT}}$ signal. The expressions $\mathrm{THb}^{\mathrm{MSOT}}$ and $\mathrm{sO}_{2}^{\mathrm{MSOT}}$ are used to emphasize that the photoacoustically determined $\mathrm{THb}$ and $\mathrm{sO}_{2}$ biomarkers are not exactly equal to the underlying parameters, since to be able to accurately resolve absolute values, knowledge of the light fluence distribution, system response, and Grüneisen parameter is required. ${ }^{43}$ For dynamic experiments, a trendline was fitted on the increasing $\mathrm{sO}_{2}$ values and the maximum value reached during the experiment and gradient of the trendline were extracted, to be compared between the species.

Raw data extracted from the ROIs were analyzed using Python (version 2.7) and MATLAB. Statistical analysis was performed using Prism (GraphPad). All data are shown as mean \pm standard error of the mean (SEM) unless otherwise stated. Unpaired two-tailed $t$-tests were performed to calculate the statistics. Pearson's rank test was performed to assess correlations between biochemical blood parameters and $\mathrm{THb}^{\mathrm{MSOT}}$. Significance is assigned for $p$-values $<0.05$.

\section{Results}

\subsection{Gene sequence Analysis Confirms Intra- and Interspecies Homogeneity of Hemoglobin Genes in Mouse and Rat}

We first assessed the intraspecies genetic correspondence of the two $\alpha$-and two $\beta$-globin chains of the HB tetramer in rat and mouse, as alterations in HB globin genes can lead to changes in the protein structure that might affect the PAI signal. We found that an intraspecies homogeneity of $100 \%$ could be determined $(n=3$, Table 1). These results indicate that intraspecies signal variations in the following experiment are unlikely to be caused by genetic missense mutations, but rather by other physiological or technical sources.

\subsection{PAI THb Correlates Directly with Biochemical HB Values Obtained Using a Hematology Analyzer and Detects Intraspecies Variations}

The relationship between the $\mathrm{THb}^{\mathrm{MSOT}}$ biomarker and biochemical blood parameters was examined. $\mathrm{THb}^{\mathrm{MSOT}}$ values from photoacoustic images taken from our static blood phantoms correlated with biochemical HB [Fig. 1(a)], HCT [Fig. 1(b)], and RBC [Fig. 1(c)] in the same mouse blood sample (HB: Pearson $r=0.6867, p=0.0047$; HCT: Pearson $r=0.6083, p=0.0161$; RBC: Pearson $r=0.5594, p=0.0302$ ). To determine whether these observations are speciesindependent, the correlation between $\mathrm{THb}^{\mathrm{MSOT}}$ and biochemical blood parameters was also studied in rat. Again, a significant correlation was found between $\mathrm{THb}^{\mathrm{MSOT}}$ and biochemical HB [Fig. 1(d)], HCT [Fig. 1(e)], and RBC [Fig. 1(f)] in rat blood (HB: Pearson $r=0.6786$, $p=0.0076$; HCT: Pearson $r=0.5363, p=0.0480$; and RBC: Pearson $r=0.5474$, $p=0.0427$ ) suggesting that PAI is directly sensitive to intraspecies variations in HB, HCT, and RBC parameters. 

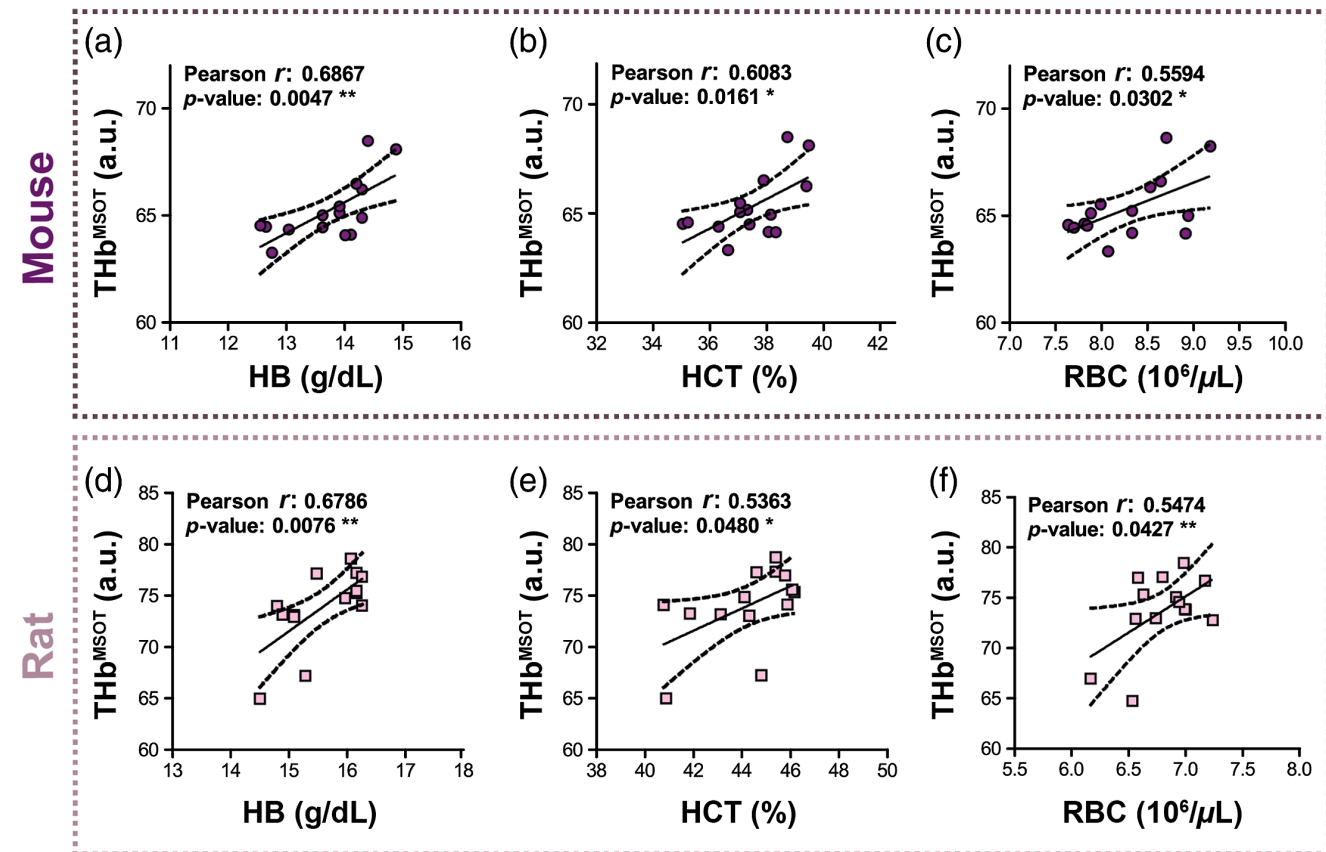

Fig. $1 \mathrm{THb}^{\mathrm{MSOT}}$ biomarker measured in tissue-mimicking phantoms corresponds to biochemical $\mathrm{HB}, \mathrm{HCT}$, and RBC levels in mouse and rat. THb $\mathrm{HSOT}^{\mathrm{M}}$ extracted from PAl data obtained from tissue-mimicking phantoms containing blood from female mice $[n=15$, purple circles, (a)-(c)] and rats $[n=14$, pink squares, (d)-(f)] correlated significantly with biochemical (a), (d) HB; (b), (e) HCT; and (c), (f) RBC count concentration in both species. ${ }^{\star} p<0.05,{ }^{* \star} p<0.01$ by Pearson correlation.

\subsection{PAI THb Resolves Interspecies Differences in HB and HCT in vitro}

After assessing the impact of intraspecies variation in biochemical blood parameters on MSOT signal, the effect of interspecies differences was analyzed. In order to exclude major differences in the protein structure and conformation yielding differences in the absorption spectra for the $\mathrm{HB}$, we first confirmed that no interspecies difference in the absorption spectra of $\mathrm{HB}$ extracted from mouse and rat blood was detected. Minor differences in total light absorbance of the samples were observed, which could be explained by different HB levels of rat and mouse blood (Table 2); thus, absorption spectra were normalized to the area under the curve for further comparison. In line with the literature, ${ }^{44,45}$ the experimentally determined spectra of mouse and rat blood demonstrated a very good agreement [Fig. 2(a)].

Next, $\mathrm{THb}^{\mathrm{MSOT}}$ was compared to the biochemical blood parameters of the different species. A significantly higher $\mathrm{THb}^{\mathrm{MSOT}}$ level was observed for the rat $[p<0.0001$, Fig. 2(b)]. Corresponding to this observation, significantly higher HB [Fig. 2(c)] and HCT [Fig. 2(d)] values could be observed in this species $(p<0.0001)$. Interestingly, the rat was characterized by

Table 2 Comparison of experimental and literature HB and HCT values of mouse $(n=15)$ and rat $(n=14)$.

\begin{tabular}{lcc}
\hline \hline Parameter & Mouse (BALB/c) & Rat (Wistar) \\
\hline HB (exp.) (g/dL) (mean \pm std) & $14.73 \pm 2.09$ & $15.06 \pm 2.35$ \\
HB (lit.) (g/dL) & 11 to 16 & 14.4 to 18.0 \\
HCT (exp.) (\%) (mean \pm std) & $41.83 \pm 6.38$ & $45.50 \pm 6.13$ \\
HCT (lit.) (\%) & 37 to 52 & 36 to 48 \\
Reference & Santos et al. $(2016)^{34}$ & Charles River Laboratories, $1998^{35}$ \\
\hline \hline
\end{tabular}


(a)
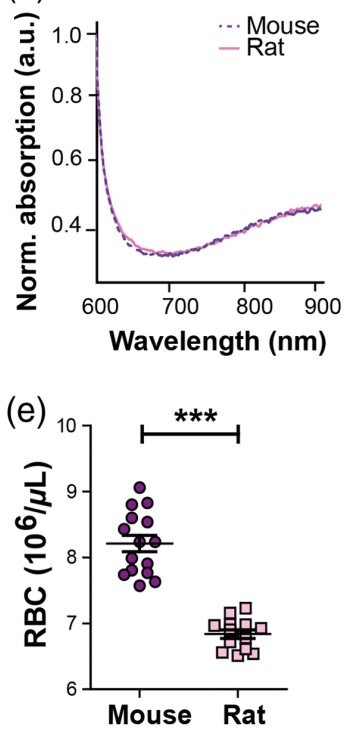

(b)
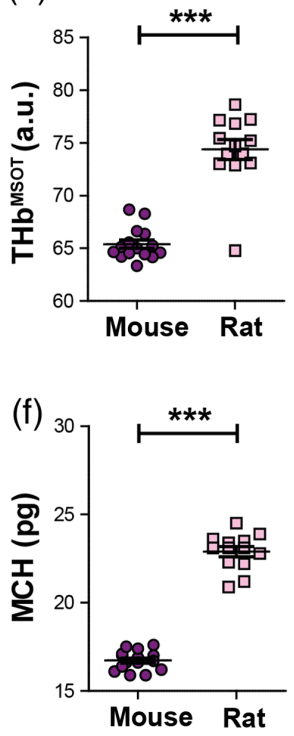

(c)
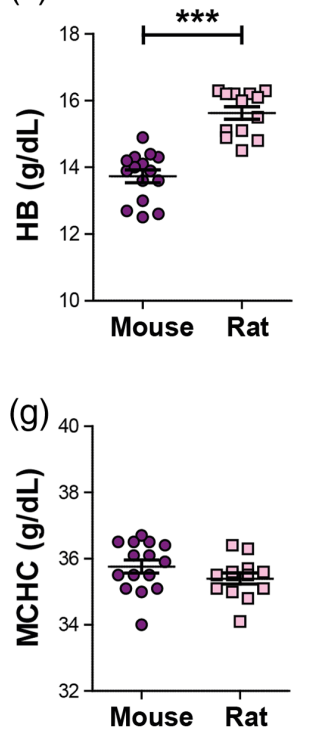

(d)

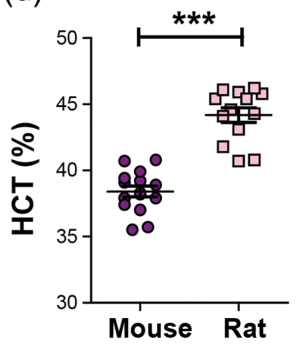

(h)

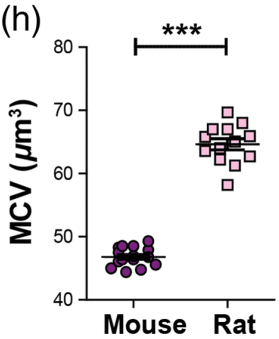

Fig. 2 PAl resolves interspecies HB differences in rat and mouse. (a) Absorption spectra of HB extracted from lysed whole blood of mouse (purple) and rat (pink). (b) Interspecies differences in $\mathrm{TH}^{\mathrm{MSOT}}$ obtained from PAI measurements of female mouse $(n=15)$ and rat $(n=14)$ blood show a significantly higher value in rat blood, which was underscored by differences in biochemical HB (c) and HCT (d). Notably, the RBC value (e) was found to be lower in the rat, but with higher mean corpuscular hemoglobin $(\mathrm{MCH})$ per red blood cell (f). While the mean corpuscular hemoglobin concentration (MCHC) was comparable between the species $(\mathrm{g})$, the mean corpuscular volume MCV (h) was higher in the rat. Data are represented as mean $\pm S E M$, significance ${ }^{\star \star \star} p<0.0001$ by unpaired $t$-test.

significantly lower RBC levels [Fig. 2(e)]. We compared the MCH levels between the two species to establish whether this observation was associated with differences in the average HB amount per RBC. In correspondence to the HB and HCT values, significant higher MCH levels were found in the rat [Fig. 2(f), $p<0.0001]$. A more detailed analysis revealed that the higher $\mathrm{MCH}$ values in the rat do not originate from a higher MCHC value [Fig. $2(\mathrm{~g})]$, but rather from a larger MCV of the RBC [ $p<0.0001$, Fig. 2(h)]. These results suggest that PAI correctly resolves interspecies differences in HB and HCT in vitro, but can only be used to quantitatively compare interspecies $\mathrm{RBC}$ values when $\mathrm{MCH}$ values are within the same range.

\subsection{PAI Resolves Interspecies Differences in Oxygenation Dynamics}

We next examined whether PAI has sufficient sensitivity to capture interspecies differences in oxygenation dynamics in mice, rat, and human blood based on the known differences in ODCs in the literature between these species [Fig. 3(a)]. ${ }^{26}$ Under standard conditions $\left[\mathrm{pH}=7.4, \mathrm{pCO}_{2}=40 \mathrm{mmHg}(5.3 \mathrm{kPa})\right.$, temperature $=37^{\circ} \mathrm{C}$, carboxyhemoglobin $\left.<2 \%\right]$, human HB is known to have the highest oxygen affinity with an ODC shifted farthest to the left $\left(\mathrm{p} 50_{\text {std }}=26 \mathrm{mmHg}\right)$, followed by rat $\left(\mathrm{p} 50_{\text {std }}=32 \mathrm{mmHg}\right)$, and then mouse HB $\left(\mathrm{p} 50_{\text {std }}=48.5 \mathrm{mmHg}\right)^{26}$ [Fig. 3(a)].

To test whether MSOT is able to qualitatively resolve these differences, fully oxygenated blood was introduced into a dynamic flow phantom system and the blood was gradually deoxygenated while the absorption spectra, $\mathrm{pO}_{2}$, temperature, and PAI spectral data were recorded in real time. The species-specific ODCs were compared by plotting $\mathrm{sO}_{2}^{\mathrm{MSOT}}$ against $\mathrm{pO}_{2}$ and showed broadly similar results as reported in the literature ${ }^{25,26}$ [Fig. 3(b)]. In a similar pattern, the human ODC was found to be shifted farthest to the left ( $550_{\mathrm{MSOT}}=29.00 \pm 2.94 \mathrm{mmHg}$ ), followed by the rat $\left(\mathrm{p} 50_{\mathrm{MSOT}}=77.75 \pm 4.99 \mathrm{mmHg}\right.$ ), and then the mouse $\left(\mathrm{p} 50_{\mathrm{MSOT}}=\right.$ $93.50 \pm 3.54 \mathrm{mmHg}$ ) [Fig. 3(c)]. These results indicate that MSOT is capable of qualitatively resolving interspecies oxygenation dynamics in vitro. 

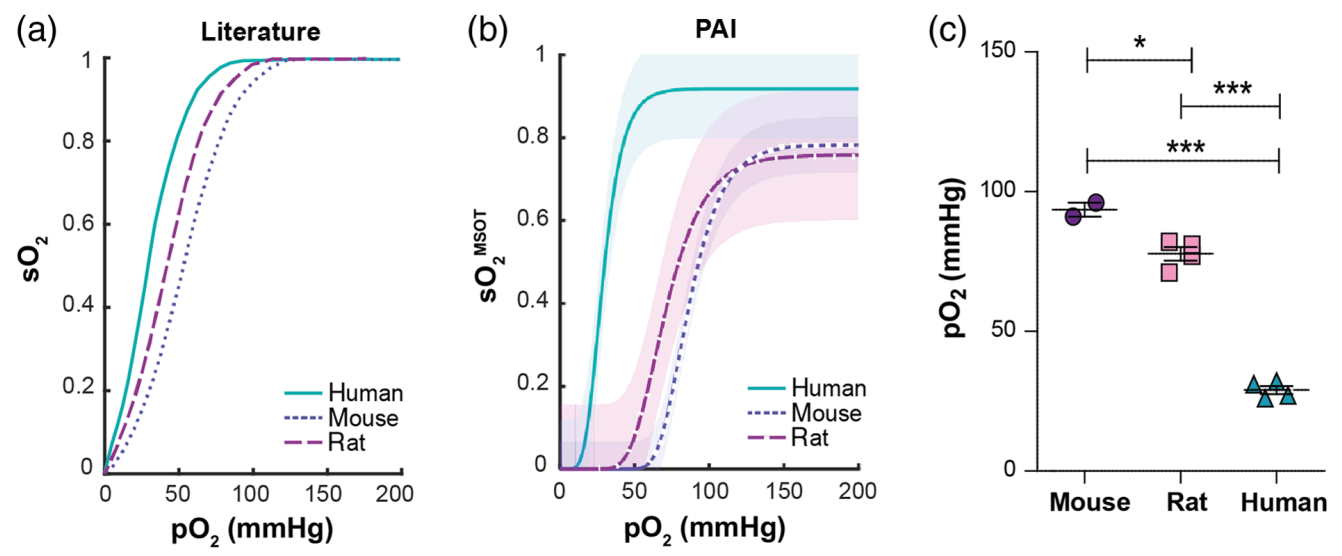

Fig. 3 PAI resolves interspecies differences in ODCs between mouse, rat, and human. Oxygenated mouse $(n=2)$, rat $(n=4)$, and human $(n=5)$ blood samples were deoxygenated in a dynamic flow phantom circuit at room temperature and changes in absorption spectrum, $\mathrm{pO}_{2}$, and PAI signals were recorded. (a) Literature values for ODCs of the respective species under standard conditions. ${ }^{26}$ (b) $\mathrm{sO}_{2}^{\mathrm{MSOT}}$ was calculated from PAl images using the absorption spectra measured within the flow phantom circuit for spectral unmixing. The resulting values were plotted against the measured $\mathrm{pO}_{2}$ of the blood within the circuit at the same time point in order to produce an ODC. (c) Extracted $\mathrm{p} 50^{\mathrm{MSOT}}$ values denoting the $\mathrm{pO}_{2}$ at $50 \% \mathrm{sO}_{2}^{\mathrm{MSOT}}$ are shown. It should be noted that the ODC influencing factors 2,3-DPG concentration, acid-base balance, and amount of dyshemoglobins were not standardized, but rather reflect the physiological values found in the respective species.

\subsection{PAl is Sensitive to the Enhanced Oxygen Dissociation Curve of the Naked Mole-Rat}

Having established the capacity for PAI to resolve differences in both HB parameters and oxygenation dynamics in mice and rats, we made a first PAI study of blood from the naked mole-rat. The naked mole-rat is adapted to live in a hypoxic underground environment, which involves having a higher affinity for oxygen in its HB molecules than rodents of similar size. ${ }^{42}$

As only small amounts of naked mole-rat blood could be obtained, a simpler experimental design was used for the studies in which small blood samples were oxygenated with $\mathrm{H}_{2} \mathrm{O}_{2}$ $(0.03 \%$ in PBS) and the oxygenation measured using PAI during $4 \mathrm{~min}$. A significantly higher maximum $\mathrm{sO}_{2}^{\mathrm{MSOT}}$ after 4 min could be found for the naked mole-rat blood in comparison to the same experiment conducted with mouse and rat blood [Fig. 4(a)]. Some deviation is observed between the maximum $\mathrm{sO}_{2}^{\mathrm{MSOT}}$ obtained for the rat in this experiment compared to the earlier findings (Fig. 3), although it is still within the bounds of error. The deviation is likely due to
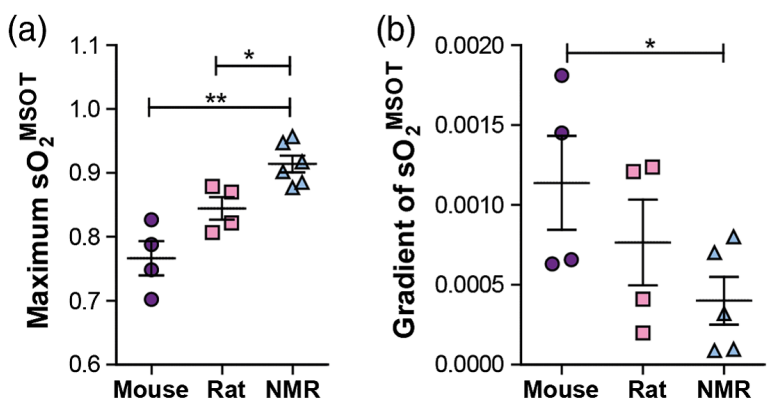

Fig. 4 PAl evaluation of oxygenation dynamics is sensitive to the higher oxygen affinity of naked mole-rat blood. Blood samples taken from mouse $(n=4)$, rat $(n=4)$, and naked mole-rat (NMR; $n=5$ ) were oxygenated using $10 \mu \mathrm{L}$ hydrogen peroxide $\left(0.03 \%\right.$ in PBS) at $37^{\circ} \mathrm{C}$. (a) The maximum $\mathrm{sO}_{2}^{\mathrm{MSOT}}$ was highest in the naked mole-rat. (b) Considering the sigmoidal shape of the ODC, this would be expected to lead to the lowest gradient of the oxygenation change, which was confirmed in our experiments. 
experiments being conducted on different batches of blood provided by an external supplier, which may have experienced different extractions or handling beyond our control. Considering the sigmoidal shape of the ODC, the highest maximum $\mathrm{sO}_{2}^{\mathrm{MSOT}}$ observed in the naked molerat should be accompanied with the lowest gradient of the oxygenation change, which was indeed found in our experiments [Fig. 4(b)]. The results suggest that PAI is able to resolve the higher oxygen affinity of the naked-mole rat blood ${ }^{42}$ compared to mouse and rat, indicated by the overall higher $\mathrm{sO}_{2}$ and lower change of $\mathrm{sO}_{2}$ when oxygenating the samples under similar experimental conditions.

\section{Discussion}

PAI holds substantial potential for application in the measurement of $\mathrm{THb}$ and $\mathrm{sO}_{2}$ biomarkers, however, our understanding of the relationship between these biomarkers and the biochemical parameters of blood including HB concentration, HCT, and ODCs has yet to be fully elucidated. Here, we sought to study the sensitivity of PAI toward physiological variations of $\mathrm{HB}$, including both intra- and interspecies variations.

Our results indicate that PAI determined $\mathrm{THb}^{\mathrm{MSOT}}$ and $\mathrm{sO}_{2}^{\mathrm{MSOT}}$ can resolve physiological variations in $\mathrm{HB}$ and HCT both within and between species. We found significant linear correlations of $\mathrm{THb}^{\mathrm{MSOT}}$ with $\mathrm{HB}, \mathrm{HCT}$, and RBC count. As HCT and HB linearly depend on each other (roughly $\mathrm{HB}=\mathrm{HCT} / 3$ ), ${ }^{46}$ it is unsurprising that both show linear trends. Our findings indicate that when the concentration of corpuscular HB is comparable between subjects, differences in RBC count could, in principle, be determined using PAI, however, this would require a low variance of the corpuscular $\mathrm{HB}$ value within the investigated group of subjects. Even within species, significant variations in $\mathrm{MCH}$ can occur due to disease-related macro- or microcytic anemia or age, ${ }^{47}$ which should be considered when making any conclusions from PAI signal to RBC number.

We could further show that differences in interspecies oxygenation dynamics based on the measurement of the PAI biomarker $\mathrm{sO}_{2}^{\mathrm{MSOT}}$ could be clearly resolved. This is notable given the diversity of species studied. In particular, our studies revealed that $\mathrm{sO}_{2}^{\mathrm{MSOT}}$ is sensitive to the differences in ODCs between mouse, rat, and human, as well as naked mole-rat. It has to be noted that our study only aimed for a qualitative comparison of the ODC curves between the species. For a quantitative comparison of ODC characteristics and p50 values, other ODC influencing factors, such as species-specific 2,3-diphosphoglycerate (2,3-DPG) concentration, ${ }^{48}$ $\mathrm{pH},{ }^{49}$ amount of dyshemoglobins, ${ }^{41}$ or the partial pressure of carbon dioxide $\left(\mathrm{pCO}_{2}\right)^{50}$ would also need to be taken into consideration.

Our study indicates that the application of PAI to detect functional differences in HB should be considered carefully when comparing data obtained from different species, particularly when using an "oxygen-enhanced" or "gas challenge" imaging protocol. ${ }^{10,51}$ It also highlights the exciting potential of PAI derived biomarkers to be applied for studies in disease-associated anaemia ${ }^{52}$ or hemoglobinopathies, such as sickle cell anemia. ${ }^{53}$ Patients with hemoglobinopathies where globin proteins are structurally abnormal often show shifts in the ODC, ${ }^{54}$ which may be captured using PAI.

Despite these promising findings, there remain some limitations of the study. We used a standard linear spectral unmixing method for resolving the contributions of $\mathrm{HbR}$ and $\mathrm{HbO}_{2}$ to our signals, which produced values of $\mathrm{sO}_{2}^{\mathrm{MSOT}}$ with limited dynamic range, particularly at the higher end of the ODCs, when compared to ground truth. Employing more advanced multispectral processing techniques should further enhance the accuracy of the photoacoustically determined $\mathrm{sO}_{2}$ estimation, however, improved spectral classification methods remain a topic of active research in the field and an optimal solution has yet to be reached. ${ }^{55-57}$

Further, our in vitro phantom experiments provide a well-controlled reference measurement for both the HB absorption spectrum and the partial pressure of oxygen. Although it may be possible in future work to use the knowledge of interspecies variations observed in this study to make a qualitative in vivo comparison of the PAI biomarker response, for further validation of our findings, in vivo confirmation would be advantageous. Unfortunately, the experimental design would be complex because of the imaging artifacts that arise due to: tissue heterogeneities, 
which lead to local variations in optical and acoustic properties, and consequently to uncertainty in PAI fluence distributions ${ }^{58,59}$ motion, such as breathing or heartbeart; ${ }^{60}$ and anatomical positioning within a nonrigid animal holder. ${ }^{36}$ Furthermore, factors influencing blood extraction, such as blood clotting, hemolysis, dilution of blood with interstitial fluid, or varying lag time before the analysis of the sample can affect the determination of the biochemical blood parameters. ${ }^{61}$ Nonetheless, such studies are of particular importance when PAI is performed in a clinical environment, as globin gene mutations in humans are common, affecting around 7\% of the overall population. ${ }^{62,63}$ Moreover, $\mathrm{HB}$ concentrations have been found to vary with human ethnic group ${ }^{64-66}$ which could impact the acquired results in studies of mixed populations.

In summary, our findings highlight the encouraging capacity of PAI to resolve intra- and interspecies differences in HB-related blood parameters and oxygenation dynamics in vitro in a sensitive and label-free manner. These results suggest promising future avenues for application of PAI for HB- and oxygenation-related research studies, strengthening the position of PAI as a powerful and versatile tool in biomedicine.

\section{Disclosures}

J. B., I. Q G., and S. E. B. have previously received research support from iThera Medical GmbH and Cyberdyne Inc.

\section{Acknowledgments}

The authors thank the Biorepository Unit (BRU, Matthew Clayton) of the CRUK Cambridge Institute for their assistance with mouse and rat blood and Dr. Doris Rassl and the Royal Papworth Hospital tissue bank for providing the human blood. This work was funded by Cancer Research UK under Grant Nos. C14303/A17197, C9545/A29580, C47594/A16267, and C197/A16465 (SEB) as well as C56829/A22053 (EStJS). L. H. is funded by a studentship from the National Physical Laboratory.

\section{Code, Data, and Materials Availability}

All associated code and raw data for this manuscript are available in the University of Cambridge repository at: https://doi.org/10.17863/CAM.55512. All materials used in the preparation of phantoms are readily available from commercial suppliers as detailed herein and further in Ref. 39.

\section{References}

1. A. G. Bell, "On the production and reproduction of sound by light," Am. J. Sci. s3-20, 305-324 (1880).

2. A. Taruttis and V. Ntziachristos, "Advances in real-time multispectral optoacoustic imaging and its applications," Nat. Photonics 9, 219-227 (2015).

3. P. Beard, Biomedical photoacoustic imaging," Interface Focus 1, 602-631 (2011).

4. G. Diot et al., "Multispectral optoacoustic tomography (MSOT) of human breast cancer," Clin. Cancer Res. 23, 6912-6922 (2017).

5. I. Quiros-Gonzalez et al., "Optoacoustics delineates murine breast cancer models displaying angiogenesis and vascular mimicry," Br. J. Cancer 118, 1098-1106 (2018).

6. M. Heijblom et al., "Photoacoustic image patterns of breast carcinoma and comparisons with magnetic resonance imaging and vascular stained histopathology," Sci. Rep. 5, 11778 (2015).

7. I. Stoffels et al., "Metastatic status of sentinel lymph nodes in melanoma determined noninvasively with multispectral optoacoustic imaging," Sci. Transl. Med. 7, 317ra199 (2015).

8. G. C. Langhout et al., "Detection of melanoma metastases in resected human lymph nodes by noninvasive multispectral photoacoustic imaging," Int. J. Biomed. Imaging 2014, 1-7 (2014). 
9. V. S. Dogra et al., "Multispectral photoacoustic imaging of prostate cancer: preliminary ex-vivo results," J. Clin. Imaging Sci. 3, 41 (2013).

10. M. R. Tomaszewski et al., "Oxygen-enhanced and dynamic contrast-enhanced optoacoustic tomography provide surrogate biomarkers of tumor vascular function, hypoxia, and necrosis," Cancer Res. 78, 5980-5991 (2018).

11. X. Yang and L. Xiang, "Photoacoustic imaging of prostate cancer," J. Innov. Opt. Health Sci. 10, 1730008 (2017).

12. W. Roll et al., "Multispectral optoacoustic tomography of benign and malignant thyroid disorders-a pilot study," J. Nucl. Med. 60(10), 1461-1466 (2019).

13. A. Rosenthal, F. A. Jaffer, and V. Ntziachristos, "Intravascular multispectral optoacoustic tomography of atherosclerosis: prospects and challenges," Imaging Med. 4, 299-310 (2012).

14. A. Taruttis et al., "Optoacoustic imaging of human vasculature: feasibility by using a handheld probe," Radiology 281, 256-263 (2016).

15. I. Ivankovic et al., "Real-time volumetric assessment of the human carotid artery: handheld multispectral optoacoustic tomography," Radiology 291, 45-50 (2019).

16. N. Bhutiani et al., "Noninvasive imaging of colitis using multispectral optoacoustic tomography," J. Nucl. Med. 58, 1009-1012 (2017).

17. A. Brown and A. L. Goodall, "Normal variations in blood haemoglobin concentration," J. Physiol. 104(4), 404-407 (1946).

18. J. W. Adamson and C. A. Finch, "Hemoglobin function, oxygen affinity, and erythropoietin," Annu. Rev. Physiol. 37, 351-369 (1975).

19. M. A. Johnson-Spear and R. Yip, "Hemoglobin difference between black and white women with comparable iron status: justification for race-specific anemia criteria," Am. J. Clin. Nutr. 60, 117-121 (1994).

20. X. Dong et al., "A population-based study of hemoglobin, race, and mortality in elderly persons," J. Gerontol. Ser. A 63, 873-878 (2008).

21. W. W. Hawkins, E. Speck, and V. G. Leonard, "Variation of the hemoglobin level with age and sex," Blood 9, 999-1007 (1954).

22. C. S. Williamson, "Influence of age and sex on hemoglobin," Arch. Intern. Med. XVIII, 505 (1916).

23. B. Vahlquist, "The cause of the sexual differences in erythrocyte hemoglobin and serum iron levels in human adults," Blood 5, 874-875 (1950).

24. D. M. Mintzer, S. N. Billet, and L. Chmielewski, "Drug-induced hematologic syndromes," Adv. Hematol. 2009, 1-11 (2009).

25. L. H. Gray and J. M. Steadman, "Determination of the oxyhaemoglobin dissociation curves for mouse and rat blood," J. Physiol. 175, 161-171 (1964).

26. K. Schmidt-Neilsen and J. L. Larimer, "Oxygen dissociation curves of mammalian blood in relation to body size," Am. J. Physiol. 195(2), 424-428 (1958).

27. B. G. Forget and H. F. Bunn, "Classification of the disorders of hemoglobin," Cold Spring Harbor perspectives in medicine 3(2), a011684 (2013).

28. T. J. Park et al., "Fructose-driven glycolysis supports anoxia resistance in the naked molerat," Science 356, 307-311 (2017).

29. A. Untergasser et al., "Primer3-new capabilities and interfaces," Nucleic Acids Res. 40, e115-e115 (2012).

30. "PCR Using Q5® Hot Start High-Fidelity DNA Polymerase (M0493)|NEB," https:// international.neb.com/protocols/2012/08/30/pcr-using-q5-hot-start-high-fidelity-dnapolymerase-m0493 (accessed 20 November 2019).

31. F. Sievers and D. G. Higgins, "Clustal omega, accurate alignment of very large numbers of sequences," in Methods in Molecular Biology, D. Russell, Ed., Vol. 1079, pp. 105-116, Humana Press, Totowa, New Jersey (2014).

32. A. K. Wassmuth et al., "Evaluation of the Mythic 18 hematology analyzer for use with canine, feline, and equine samples," J. Vet. Diagn. Invest. 23, 436-453 (2011).

33. S. F. Russo and R. B. Sorstokke, "Hemoglobin. Isolation and chemical properties," J. Chem. Educ. 50, 347-350 (1973). 
34. E. W. Santos et al., "Valores de referência hematológicos e bioquímicos para camundongos das linhagens C57BL/6, Swiss Webster e BALB/c," Braz. J. Veterinary Res. Anim. Sci. 53(2), 138-145 (2016).

35. Charles River Laboratories, "Baseline hematology and clinical chemistry values for Charles River Wistar Rat.," https://www.criver.com/sites/default/files/resources/Baseline HematologyandClinicalChemistryValuesforCharlesRiverWistarRats\%5BCrlWIBR\%5Dasa FunctionofSexandAgeSpring 1998.pdf (1998).

36. J. Joseph et al., "Evaluation of precision in optoacoustic tomography for preclinical imaging in living subjects," J. Nucl. Med. 58, 807-814 (2017).

37. S. Morscher et al., "Semi-quantitative multispectral optoacoustic tomography (MSOT) for volumetric PK imaging of gastric emptying," Photoacoustics 2, 103-110 (2014).

38. A. Dima, N. C. Burton, and V. Ntziachristos, "Multispectral optoacoustic tomography at 64, 128, and 256 channels," J. Biomed. Opt. 19, 036021 (2014).

39. M. Gehrung, S. E. Bohndiek, and J. Brunker, "Development of a blood oxygenation phantom for photoacoustic tomography combined with online $\mathrm{pO}_{2}$ detection and flow spectrometry," J. Biomed. Opt. 24, 121908 (2019).

40. B. K. McNab, "The metabolism of fossorial rodents: a study of convergence," Ecology 47, 712-733 (1966).

41. S. Patel and S. S. Mohiuddin, Physiology, Oxygen Transport and Carbon Dioxide Dissociation Curve, StatPearls Publishing, Treasure Island, Florida (2020).

42. K. Johansen et al., "Blood respiratory properties in the naked mole rat Heterocephalus glaber, a mammal of low body temperature," Respir. Physiol. 28, 303-314 (1976).

43. B. Cox et al., "Quantitative spectroscopic photoacoustic imaging: a review," J. Biomed. Opt. 17, 061202 (2012).

44. D. A. Grosenbaugh, J. O. Alben, and W. W. Muir, "Absorbance spectra of inter-species hemoglobins in the visible and near infrared regions," J. Vet. Emergency Crit. Care 7, 36-42 (1997).

45. W. G. Zijlstra et al., "Spectrophotometry of hemoglobin: absorption spectra of rat oxyhemoglobin, deoxyhemoglobin, carboxyhemoglobin, and methemoglobin," Comp. Biochem. Physiol. Part B 107, 161-166 (1994).

46. L. Quintó et al., "Relationship between haemoglobin and haematocrit in the definition of anaemia," Trop. Med. Int. Health 11, 1295-1302 (2006).

47. T. I. Restell et al., "Hematology of Swiss mice (Mus musculus) of both genders and different ages," Acta Cirurgica Bras. 29, 306-312 (2014).

48. R. Macdonald, "Red cell 2,3-diphosphoglycerate and oxygen affinity," Anaesthesia 32, 544-553 (1977).

49. G. S. Adair, "The hemoglobin system. VI. The oxygen dissociation curve of hemoglobin," J. Biol. Chem. 63, 529-545 (1925).

50. R. M. Winslow et al., "Simulation of continuous blood O2 equilibrium curve over physiological pH, DPG, and PCO2 range," J. Appl. Physiol. 54, 524-529 (1983).

51. M. R. Tomaszewski et al., "Oxygen enhanced optoacoustic tomography (OE-OT) reveals vascular dynamics in murine models of prostate cancer," Theranostics 7, 2900-2913 (2017).

52. W.-P. Pan, Y.-H. Teng, and J. Shen, "Photoacoustic study of iron-deficiency anaemia," J. Biomed. Eng. 13, 415-416 (1991).

53. C. Cai et al., "Photoacoustic flow cytometry for single sickle cell detection in vitro and in vivo," Anal. Cell. Pathol. 2016, 1-11 (2016).

54. J.-A. Collins et al., "Relating oxygen partial pressure, saturation and content: the haemoglobin-oxygen dissociation curve," Breathe 11, 194-201 (2015).

55. S. Tzoumas et al., "Eigenspectra optoacoustic tomography achieves quantitative blood oxygenation imaging deep in tissues," Nat. Commun. 7, 12121 (2016).

56. J. Gröhl et al., "Estimation of blood oxygenation with learned spectral decoloring for quantitative photoacoustic imaging (LSD-qPAI)," Med. Phys., https://arxiv.org/abs/1902.05839 (2019).

57. S. Tzoumas and V. Ntziachristos, "Spectral unmixing techniques for optoacoustic imaging of tissue pathophysiology," Philos. Trans. R. Soc. A 375, 20170262 (2017). 
58. B. Liu et al., "Phantom and in-vivo measurements of hemoglobin concentration and oxygen saturation using PCT-S small animal scanner," Proc. SPIE 6437, 64371X (2007).

59. B. T. Cox, J. G. Laufer, and P. C. Beard, "The challenges for quantitative photoacoustic imaging," Proc. SPIE 7177, 717713 (2009).

60. X. L. Deán-Ben and D. Razansky, "Adding fifth dimension to optoacoustic imaging: volumetric time-resolved spectrally enriched tomography," Light Sci. Appl. 3, e137-e137 (2014).

61. L. Berkow, "Factors affecting hemoglobin measurement," J. Clin. Monit. Comput. 27, 499-508 (2013).

62. E. Kohne, "Hemoglobinopathies," Dtsch. Arztebl. Int. 108, 532-540 (2011).

63. D. J. Weatherall and J. B. Clegg, "Inherited haemoglobin disorders: an increasing global health problem," Bull. World Health Organ. 79, 704-12 (2001).

64. J. L. Cresanta et al., "Racial difference in hemoglobin concentration of young adults," Prev. Med. 16, 659-669 (1987).

65. D. M. Williams, "Racial differences of hemoglobin concentration: measurements of iron, copper, and zinc," Am. J. Clin. Nutr. 34, 1694-1700 (1981).

66. X. Dong et al., "A population-based study of hemoglobin, race, and mortality in elderly persons," J. Gerontol. A 63, 873-878 (2008).

Lina Hacker received her bachelor's degree in molecular biomedicine at the University of Bonn in Germany and her master's degree in biomedical engineering at RWTH Aachen in Germany. Currently, she is pursuing a PhD in Medical Sciences at the University of Cambridge focussing on the technical validation of optoacoustic imaging systems.

Biographies of the other authors are not available. 